\title{
Docking Study of Flavonols and Human c-Jun N-terminal Kinase 1
}

\author{
Jee-Young Lee, Ki-Woong Jeong, Yong Seok Heo, ${ }^{\dagger}$ and Yangmee Kim* \\ Department of Bioscience and Biotechnology, Bio/Molecular Informatics Center, Konkuk University, Seoul 143-701, Korea \\ *E-mail:ymkim@konkuk.ac.kr. \\ ${ }^{\dagger}$ Department of Chemistry, Konkuk University, Seoul 143-701, Korea \\ Received June 21, 2010, Accepted June 24, 2010
}

\begin{abstract}
c-Jun N-terminal kinase 1 (JNK1) is involved in apoptosis, cell differentiation and proliferation. It has been reported that a flavonol, quercetin, induces cell apoptosis and JNK inhibition. In order to understand the interactions of quercetin and JNK1, we performed receptor-oriented pharmacophore based in silico screening and determined a binding model of human JNK1 and quercetin at the ATP binding site of JNK1. 5-OH of A-ring and carbonyl oxygen of C-ring of quercetin participated in hydrogen bonding interactions with backbone of E109 and M111. Additionally, 3'-OH of quercetin formed a hydrogen bond with backbone of I32. One hydrophobic interaction is related on the binding of quercetin to JNK1 with I32, N114, and V158. Based on this model, we conducted a docking study with other 8 flavonols to find possible flavonoids inhibitors of JNK1. We proposed that one flavonols, rhamnetin, can be a potent inhibitor of JNK and 5-OH of A-ring and 3'-OH of B-ring of flavonols are the essential features for JNK1 inhibition.
\end{abstract}

Key Words: JNK1, Flavonoid, Apoptosis, In silico screening, Docking

\section{Introduction}

c-Jun N-terminal kinases (JNKs) are a group of mitogenactivated protein kinases (MAPKs) which stress-activated kinases. JNKs bind and phosphosphorylate c-Jun on Ser63 and Ser73 within its transcriptional activation domain. ${ }^{1-3} \mathrm{JNKs}$ have ten isoforms derived from three genes: JNK1, JNK2 and JNK3 in mammalian. ${ }^{4} \mathrm{JNK} 1$ and JNK2 are found in all cells and tissues of mammalians and JNK3 is found mainly in the brain. ${ }^{5}$ The JNK pathway is related on immune system and JNKs play a central role in obesity and insulin resistance. ${ }^{6,7}$ They are also important signaling proteins involved in apoptosis, cell differentiation and proliferation. ${ }^{1,2}$ The JNKs mediate cell stress responses as regulation of proapoptotic death signaling events. ${ }^{8-10}$ Specially, it is known that JNK1 is involved in apoptosis, cell differentiation and proliferation, and neurodegeneration. ${ }^{11}$ Therefore, inhibition of JNK may provide clinical benefits in diverse diseases and this is why JNK inhibitors have been discovered and characterized from many researchers.

Flavonoids are common constituents of plants and are widely used in medicine for treatment of several human diseases. ${ }^{12-14}$ It has been reported that quercetin, the most abundant flavonol, inhibit JNK1 and induces apoptosis. It inhibits the phosphorylation of JNK $1 .{ }^{15}$ Flavonoids show inhibitory activity against several kinases and the X-ray complex structure of kinase and some flavonoids or flavonoid derived compounds have been released. ${ }^{16,17}$

In this study, we performed receptor-oriented pharmacophore based in silico screening in order to determine the binding model between quercetin and JNK1. To find new flavonoid inhibitor of JNK1, we conducted docking study with known eight flavonols. This study will be helpful to understand the mechanism of flavonol against kinase.

\section{Methods}

Receptor-oriented pharmacophore-based in silico screening. We compared several X-ray complex structures of inhibitorJNK1 at the center of the ATP binding site. Then, we defined specific interactions between JNK1 and its inhibitors. Based on this information, we determined three pharmacophore maps and we searched a single compound library which included only quercetin. Computations were performed on a Linux environment using the DS Modeling 2.1 (Accelrys Inc., San Diego, CA).

Docking study. Based on the binding model between quercetin and JNK1, determined by in silico screening, we performed an automated docking study in order to find other possible flavonoid inhibitors of JNK1 using AutoDock. ${ }^{18-20}$ The Lamarckian Genetic Algorithm (LGA) of the AutoDock 3.05 was used for docking experiments. MD simulations on the final docking structure were performed in the canonical ensemble (NVT) at $300 \mathrm{~K}$ and distance-dependent function of the dielectric constant was used for the calculation of the energetic maps in vacuum system using the program InsightII/Discover. All atoms of the system were considered explicitly, and their interactions were computed using the consistent valence force field. A distance cutoff of $10 \AA$ was used for van der Waals interactions and electrostatic interactions. The time step in the MD simulations was $1 \mathrm{fs}$ and MD simulation was performed for 2 ns. Coordinates were saved every 1 ps. The average structure was calculated for the 2 ns trajectory and submitted to final energy minimization by performing 20,000 steps of steepest descent method.

The scoring functions (LigScore and PLP1) were calculated for hits to evaluate the accuracy of docking study using DS Modeling 2.1. 


\section{Results and Discussion}

To compare the interaction model between inhibitors and human JNK1, we superimposed several X-ray complex structures of human JNK1 and its inhibitors (1JNK.pdb, 1UKI.pdb, 2H96.pdb, 3ELJ.pdb, and 3KVX.pdb). ${ }^{3,21-24}$ Overall structure of JNK 1 and the superimposed model of five JNK1 inhibitors are represented in Figure 1. JNK1 inhibitors formed commonly two hydrogen bonds with backbone amide proton of M111 and backbone carbonyl oxygen of E109, respectively. Also, they have two possible hydrophobic interactions at the ATP binding site as shown in Figure 2. V40 and L168 form the first hydrophobic site and I32, N114, and V158 form the second hydrophobic site. On the basis of these interactions, we defined two pharmacophore maps (Map I and II). Each map consisted of
(A)

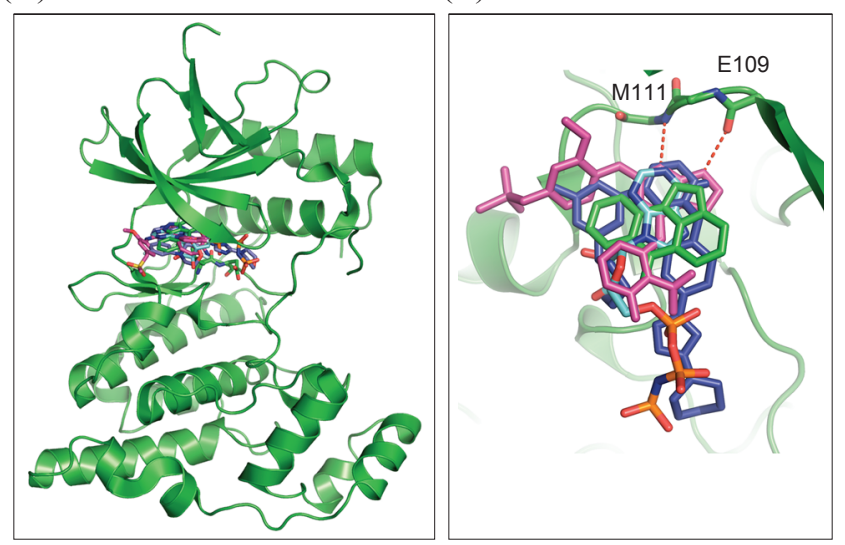

Figure 1. Interaction models of (A) Overall structure of human JNK1. (B) five hVEGFR2 inhibitors and hVEGFR2 taken from X-ray complex structures (1JNK.pdb, 1UKI.pdb, 2H96.pdb, 3ELJ.pdb, and $3 \mathrm{KVX} . \mathrm{pdb}$ ). Red dotted line presented hydrogen bonds between receptor and inhbitiors.

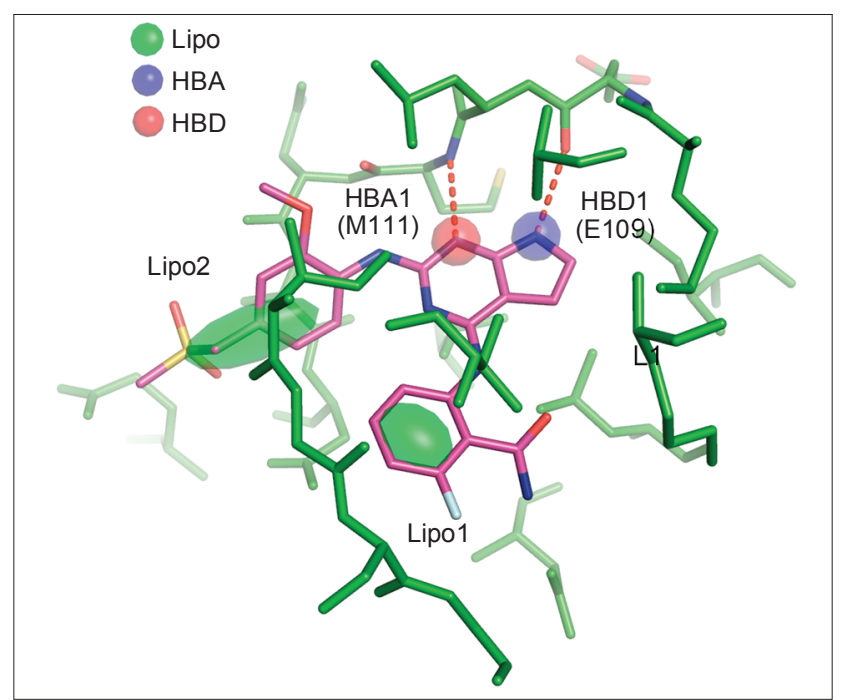

Figure 2. Representation of the interactions essential for inhibition of JNK1. HBA is hydrogen bonding acceptor, HBD is hydrogen bonding donor, and Lipo is hydrophobic interaction. three features; one hydrogen bond acceptor (HBA) on M111, one hydrogen bond donor (HBD) on E109, and one hydrophobic interaction (Lipo1 or 2). We searched the multiple conformer library of quercetin and it hit only by map I. Map I include Lipo 2 which presented the second hydrophobic site. 5-OH and carbonyl oxygen of C-ring of quercetin participated in hydrogen bonding interactions with amide proton of M111 and carbonyl oxygen of E109, respectively. It is well known that these interactions are essential between the inhibitors and JNK1. B-ring of quercetin formed a hydrophobic interaction with I32, N114 and V158. Specially, additional hydrogen bonding interaction was found between the backbone carbonyl oxygen of I32 and 3'-OH of quercetin. This is an exclusive interaction found only in binding model of quercetin and JNK1.

To find possible flavonoid inhibitors of JNK1, we conducted docking study based on the binding model of quercetin and JNK1. Since quercetin is classified as flavonol and we once evaluated the possibility of flavonols as kinase inhibitors in our previous report, we used the set of 8 flavonols for docking study. ${ }^{25}$ Flavonols are a class of flavonoids and present in a wide variety of vegetables and fruits.

Among eight flavonols, five (kaempferol, rhamnetin, kaempferide, galangin, and morin) were hit as candidates of JNK1 inhibitors. Five hits have 3-, 5-, and 7-OH. 5-OH formed a hydrogen bond with backbone oxygen of E109. Also, they have two hydroxyl groups at the B-ring at 3'- and 4'-position. Interestingly, 3'-OH of quercetin participated in hydrogen bonding

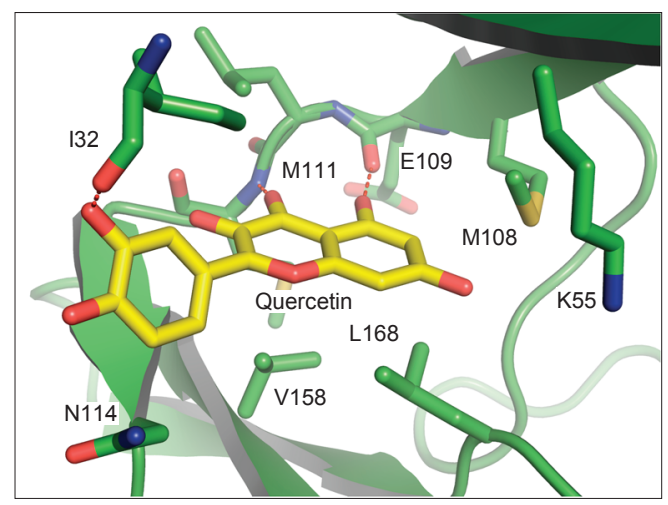

(B)

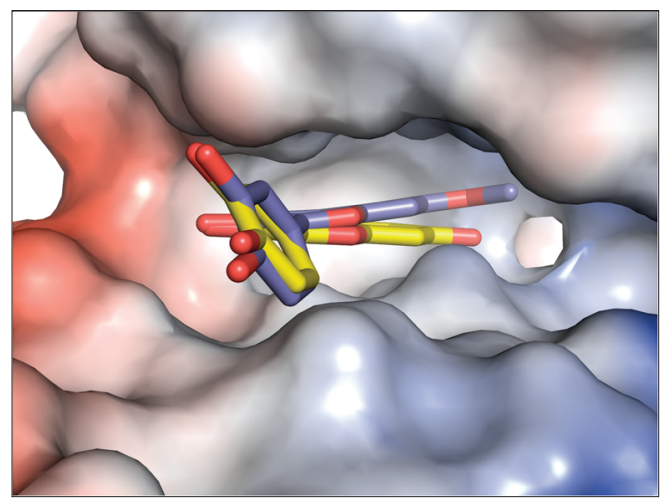

Figure 3. (A) Binding model of quercetin and JNK1 by in silico screening. Red dotted line presented hydrogen bonds between receptor and inhbitiors. (B) The superimposed surface model of quercetin (yellow) and rhamnetin (blue) with JNK1. 
Table 1. Structures of flavonols based on the position of their substituents and scoring functions of hit

$$
\text { (1) }
$$

\begin{tabular}{|c|c|c|c|c|c|c|c|c|c|}
\hline \multirow{2}{*}{ Flavonols } & \multicolumn{7}{|c|}{ Position of Substituents } & \multirow{2}{*}{ LigScore } & \multirow{2}{*}{ PLP1 } \\
\hline & 3 & 5 & 7 & $2^{\prime}$ & $3^{\prime}$ & $4^{\prime}$ & $5^{\prime}$ & & \\
\hline Quercetin & $\underline{\mathrm{OH}}$ & $\underline{\mathrm{OH}}$ & $\underline{\mathrm{OH}}$ & $\underline{\mathrm{H}}$ & $\underline{\mathrm{OH}}$ & $\underline{\mathrm{OH}}$ & $\underline{\mathrm{H}}$ & 2.89 & $\underline{62.61}$ \\
\hline Galangin & $\overline{\mathrm{OH}}$ & $\overline{\mathrm{OH}}$ & $\overline{\mathrm{OH}}$ & $\overline{\mathrm{H}}$ & $\overline{\mathrm{H}}$ & $\overline{\mathrm{H}}$ & $\overline{\mathrm{H}}$ & $-\overline{3.03}$ & $\overline{43.01}$ \\
\hline Kaempferol & $\mathrm{OH}$ & $\mathrm{OH}$ & $\mathrm{OH}$ & $\mathrm{H}$ & $\mathrm{H}$ & $\mathrm{OH}$ & $\mathrm{H}$ & -0.51 & 53.44 \\
\hline Myricetin & $\mathrm{OH}$ & $\mathrm{OH}$ & $\mathrm{OH}$ & $\mathrm{H}$ & $\mathrm{OH}$ & $\mathrm{OH}$ & $\mathrm{OH}$ & - & - \\
\hline Fisetin & $\mathrm{OH}$ & $\mathrm{H}$ & $\mathrm{OH}$ & $\mathrm{H}$ & $\mathrm{OH}$ & $\mathrm{OH}$ & $\mathrm{H}$ & - & - \\
\hline Morin & $\mathrm{OH}$ & $\mathrm{OH}$ & $\mathrm{OH}$ & $\mathrm{OH}$ & $\mathrm{H}$ & $\mathrm{OH}$ & $\mathrm{H}$ & -8.25 & 25.93 \\
\hline Kaempferide & $\mathrm{OH}$ & $\mathrm{OH}$ & $\mathrm{OH}$ & $\mathrm{H}$ & $\mathrm{H}$ & $\mathrm{O}-\mathrm{Me}$ & $\mathrm{H}$ & 0.63 & 52.39 \\
\hline Rhamnetin & $\mathrm{OH}$ & $\mathrm{OH}$ & $\mathrm{O}-\mathrm{Me}$ & $\mathrm{H}$ & $\mathrm{OH}$ & $\mathrm{OH}$ & $\mathrm{H}$ & 2.54 & 64.14 \\
\hline$\overline{\text { Pachypodol }}$ & $\overline{\mathrm{O}-\mathrm{Me}}$ & $\overline{\mathrm{OH}}$ & $\overline{\mathrm{O}-\mathrm{Me}}$ & $\overline{\mathrm{H}}$ & $\overline{\mathrm{O}-\mathrm{Me}}$ & $\overline{\mathrm{OH}}$ & $\overline{\mathrm{H}}$ & - & - \\
\hline
\end{tabular}

-O-Me = Methoxy group

interaction with backbone of Ile 32 and this would be the essential feature of flavonols to inhibit JNK1. Among the non-hits, fisetin does not have 5-OH and two methoxy groups of pachypodol may interrupt formation of hydrogen bonding of 5-OH. In the case of myricetin, the extra hydroxyl group of 5'-position may cause steric conflict with side chain of receptor.

In order to verify the results of the docking study, we calculated two scoring functions; LigScore and PLP1. The LigScore possesses high predictive accuracy of affinity of ligand-receptor binding as well as $p K_{i}$ values. ${ }^{26}$ The PLP1 (Piecewise Linear Potential 1) is an empirical scoring function and the higher PLP score indicates the stronger binding affinity with the receptor. ${ }^{27}$ The 2D structures of 9 flavonols (including quercetin) and scoring values of hits are listed in Table 1.

A reference ligand, quercetin, is given a LigScore value of 2.89 and PLP1 value of 62.61. Only rhamnetin showed similar range of scoring values compared with quercetin. This means rhamnetin can be a potential inhibitor of JNK1 with similar binding affinity of quercetin. The rest of four hits showed much lower values for both scoring functions than rhamnetin.

Quercetin has five hydroxyl groups at the position 3, 5, 7, 3', and 4'. Among these, 5- and 3'-OH participated in hydrogen bonding interactions with JNK1 and these interactions can be important in its inhibitory activity against JNK1. Rhamnetin has four hydroxyl groups $\left(3,5,3^{\prime}\right.$ and $\left.4^{\prime}\right)$ and 7-position is O-methylated. 5- and 3'-OH of rhamnetin also formed hydrogen bonds with JNK1. Because 7-OH of quercetin does not have interactions with JNK1 directly, it can be possible that the methoxy group of rhamnetin does not effect on the binding affinity. Interaction model between quercetin and JNK1 and the superimposed surface model of quercetin and rhamnetin with JNK1 are depicted in Figure 3.

The rest of four hit flavonols (galangin, kaempferol, morin, and kaempferide) had very low scoring values; LigScore was less than 1 and also PLP1 was less than 55, owing to the absence of 3 '-OH. The lowest ranked flavonol, morin, has 2'-OH. Since this hydroxyl group is exposed to the surface, binding affinity of morin might be much lower than the other hits.

From the results, we can conclude that 5- and 3'-OH of flavonols are essential for binding to JNK1. Rhamnetin may be a potent JNK1 inhibitor with good binding affinities. Antiproliferative activity of rhamnetin is well known against various cell lines. ${ }^{28}$ Therefore, in our further study, we will measure the binding affinity of rhamnetin for human JNK1 and investigate the mechanism of rhamnetin.

\section{Conclusion}

We determined two pharmacophore maps which represented the specific interactions between known inhibitors and JNK1. This map consisted of three features; one HBA, one HBD, and one Lipo. We performed receptor-oriented pharmacophore based in silico screening for quercetin with two maps and determined binding model between quercetin and JNK1. 5-OH and carbonyl oxygen of C-ring of quercetin participated in hydrogen bonding interactions with backbone of M111 and E109, respectively. B-ring of quercetin formed a hydrophobic interaction with I32, N114, and V158. Additionally, 3'-OH of quercetin formed the third hydrogen bond with backbone oxygen of I32. These interactions are the characteristics of quercetin to inhibit the activity of JNK1. On the basis of this model, we conducted docking study with known eight flavonols to find new flavonoid inhibitors of JNK1 and we proposed that rhamnetin can be a potent inhibitor of JNK1 with similar binding affinity of quercetin based on two scoring functions (LigScore and PLP1). 5-OH and 3'-OH of flavonols would be the important features to inhibit of JNK1. This study may provide a strategy for the development of natural flavonoid inhibitors of JNK1.

Acknowledgments. This work was supported by Priority Research Centers Program through the National Research Foundation of Korea (NRF) funded by the Ministry of Education, 
Science and Technology (2009-0093824) and by the Korea Polar Research Institute (KOPRI PG10010). Ki-Woong Jeong is supported, in part, by the second BK21 (MOE).

\section{References}

1. Oltmanns, U.; Issa, R.; Sukkar, M. B.; John, M.; Chung, K. F. Br. J. Pharmacol. 2003, 139, 1228.

2. Bogoyevitch, M. A.; Ngoei, K. R.; Zhao, T. T.; Yeap, Y. Y.; Ng, D. C. Biochim. Biophys. Acta 2010, 1804, 463.

3. Heo, Y. S.; Kim, S. K.; Seo, C. I.; Kim, Y. K.; Sung, B. J.; Lee, H. S.; Lee, J. I.; Park, S. Y.; Kim, J. H.; Hwang, K. Y.; Hyun, Y. L.; Jeon, Y. H.; Ro, S.; Cho, J. M.; Lee, T. G.; Yang, C. H. EMBO J. 2004, 23, 2185.

4. Waetzig, V.; Herdegen, T. Trends Pharmacol. Sci. 2005, 26, 455.

5. Bode, A. M.; Dong, Z. Mol. Carcinog. 2007, 46, 591.

6. Hirosumi, J.; Tuncman, G.; Chang, L.; Görgün, C. Z.; Uysal, K. T.; Maeda, K.; Karin, M.; Hotamisligil, G. S. Nature 2002, 420, 333

7. Waeber, G.; Delplanque, J.; Bonny, C.; Mooser, V.; Steinmann, M.; Widmann, C.; Maillard, A.; Miklossy, J.; Dina, C.; Hani, E. H.; Vionnet, N.; Nicod, P.; Boutin, P.; Froguel, P. Nat. Genet. 2000, 24,291

8. Xia, Z.; Dickens, M.; Raingeaud, J.; Davis, R. J.; Greenberg, M. E. Science 1995, 270, 1326

9. Park, J.; Kim, I.; Oh, Y. J.; Lee, K.; Han, P. L.; Choi, E. J. Biol. Chem. 1997, 272, 16725.

10. Tournier, C.; Hess, P.; Yang, D. D.; Xu, J.; Turner, T. K.; Nimnual, A.; Bar-Sagi, D.; Jones, S. N.; Flavell, R. A.; Davis, R. J. Science 2000, 288, 870.

11. Hibi, M.; Lin, A.; Smeal, T.; Minden, A.; Karin, M. Genes Dev. 1993, 7, 2135

12. Leone, M.; Zhai, D.; Sareth, S.; Kitada, S.; Reed, J. C.; Pellecchia, M. Cancer Res. 2003, 63, 8118.

13. Khan, N.; Afaq, F.; Saleem, M.; Ahmad, N.; Mukhtar, H. Cancer Res. 2006, 66, 2500

14. Chung, J. Y.; Huang, C.; Meng, X.; Dong, Z.; Yang, C. S. Cancer Res. 1999, 59, 4610

15. Perez-Vizcaino, F.; Bishop-Bailley, D.; Lodi, F.; Duarte, J.; Co- golludo, A.; Moreno, L.; Bosca, L.; Mitchell, J. A.; Warner, T. D. Biochem Biophys. Res. Commun. 2006, 346, 919.

16. Lu, H.; Chang, D. J.; Baratte, B.; Meijer, L.; Schulze-Gahmen, U. J. Med. Chem. 2005, 48, 737

17. Baumli, S.; Lolli, G.; Lowe, E. D.; Troiani, S.; Rusconi, L.; Bullock, A. N.; Debreczeni, J. E.; Knapp, S.; Johnson, L. N. EMBO J. 2008, 27, 1907.

18. Lee, J. Y.; Baek, S.; Kim, Y. Bull. Korean Chem. Soc. 2007, 28, 379 .

19. Morris, G. M.; Goodsell, D. S.; Halliday, R. S.; Huey, R.; Hart, W. E.; Belew, R. K.; Olson, A. J. J. Computational Chemistry 1998, 19, 1639.

20. Lee, J. Y.; Jeong, K. W.; Kim, Y. Bull. Korean Chem. Soc. 2009, $30,1835$.

21. Xie, X.; Gu, Y.; Fox, T.; Coll, J. T.; Fleming, M. A.; Markland, W.; Caron, P. R.; Wilson, K. P.; Su, M. S. Y. Structure 1998, 6, 983.

22. Zhao, H.; Serby, M. D.; Xin, Z.; Szczepankiewicz, B. G.; Liu, M.; Kosogof, C.; Liu, B.; Nelson, L. T.; Johnson, E. F.; Wang, S.; Pederson, T.; Gum, R. J.; Clampit, J. E.; Haasch, D. L.; AbadZapatero, C.; Fry, E. H.; Rondinone, C.; Trevillyan, J. M.; Sham, H. L.; Liu, G. J. Med. Chem. 2006, 49, 4455.

23. Chamberlain, S. D.; Redman, A. M.; Wilson, J. W.; Deanda, F.; Shotwell, J. B.; Gerding, R.; Lei, H.; Yang, B.; Stevens, K. L.; Hassell, A. M.; Shewchuk, L. M.; Leesnitzer, M. A.; Smith, J. L.; Sabbatini, P.; Atkins, C.; Groy, A.; Rowand, J. L.; Kumar, R.; Mook, R. A., Jr.; Moorthy, G.; Patnaik, S. Bioorg. Med. Chem. Lett. 2009, 19, 360

24. Kamenecka, T.; Jiang, R.; Song, X.; Duckett, D.; Chen, W.; Ling, Y. Y.; Habel, J.; Laughlin, J. D.; Chambers, J.; Figuera-Losada, M.; Cameron, M. D.; Lin, L.; Ruiz, C. H.; LoGrasso, P. V. J Med Chem. 2010, 53, 419.

25. Lee, J. Y.; Jeong, K. W.; Kim, W.; Kim, Y. Bull. Korean Chem. Soc. 2009, 30, 2086.

26. Krammer, A.; Kirchhoff, P. D.; Venkatachalam, X. J. C. M.; Waldman, M. J. Mol. Graph. Model. 2005, 23, 395.

27. Verkhivker, G. M.; Bouzida, D.; Gehlhaar, D. K.; Rejto, P. A.; Arthurs, S.; Colson, A. B.; Freer, S. T.; Larson, V.; Lutyi, B. A.; Marrone, T.; Rose, P. W. Journal of Computer-Aided Molecular Design 2000, 14, 731.

28. Manthey, J. A.; Guthrie, N. J. Agric. Food Chem. 2002, 50, 5837. 